\title{
High-energy limit of mass-suppressed amplitudes in gauge theories
}

\author{
Tao Liu ${ }^{a}$ and Alexander Penin ${ }^{a, b}$ \\ ${ }^{a}$ Department of Physics, University of Alberta, \\ Edmonton AB T6G 2J1, Canada \\ ${ }^{b}$ Institute for Theoretical Physics, ETH Zürich, \\ 8093 Zürich, Switzerland \\ E-mail: 1tao@ualberta.ca, penin@itp.phys.ethz.ch
}

ABSTRACT: We present a detailed analysis of the factorization and all-order resummation of the double-logarithmic radiative corrections which determine the asymptotic behavior of the gauge theory amplitudes suppressed by the leading power of the fermion mass in the limit of high-energy fixed-angle scattering. The result is applied to estimate the bottom quark mediated contribution to the Higgs boson production in gluon fusion.

KEYwORDS: Perturbative QCD, Resummation

ARXIV EPRINT: 1809.04950 


\section{Contents}

1 Introduction 1

2 Massive quark scattering by a gluon field operator 2

2.1 The leading-order amplitude 2

2.2 Factorization of the double-logarithmic corrections 3

2.3 Resummation of the double-logarithmic corrections and the asymptotic behavior of the amplitude

2.4 Explicit evaluation of the three-loop amplitude

3 Higgs boson production mediated by bottom quark loop

4 Quark form factors beyond the leading-power approximation

$\begin{array}{lll}4.1 & \text { Vector form factor } & 14\end{array}$

$\begin{array}{ll}4.2 \text { Scalar form factor } & 18\end{array}$

$\begin{array}{llr}5 & \text { Summary and discussion } & 20\end{array}$

\section{Introduction}

A distinct feature of the gauge theory scattering amplitudes in the high-energy limit is the presence of the "Sudakov" radiative corrections enhanced by the second power of the large logarithm of the energy ratio to a characteristic infrared scale of the process per each power of the coupling constant. These double-logarithmic corrections determine the leading deviation in the asymptotic behavior of the quantum field theory amplitudes from the classical result. Since the original work [1] on the double-logarithmic approximation of the electron form factor in QED the analysis has been extended to nonabelian gauge theories and to subleading logarithms [2-7]. Sudakov logarithms exponentiate and result in a strong universal suppression of the scattering amplitudes in the limit when all the kinematic invariants of the process are large. This analysis however does not extend to the amplitudes suppressed by a power of an infrared scale in the high energy limit. The power-suppressed contributions now attract a lot of attention in various contexts (see e.g. [8-28]). Incorporating the logarithmically enhanced power-suppressed terms can significantly increase the accuracy and extend the region where the leading-power approximation is applicable. Besides their phenomenological importance these contributions are very interesting from the general effective field theory point of view since the structure of the renormalization group evolution in this case becomes highly nontrivial already in the leading logarithmic approximation.

We focus on the double-logarithmic corrections to the amplitudes suppressed by the leading power of the fermion mass. In general very little is known so far about the all-order 
structure of such corrections. In contrast to Sudakov logarithms they do not exponentiate and do not factorize into the wave functions of scattering particle. While the mass effects on the leading-power contributions have been extensively studied in the context of the high-order electroweak and QED radiative corrections [29-37], a few known examples of the all-order resummation for the power-suppressed terms are restricted to abelian gauge theory $[14,16,38,39]$. Extension of the analysis to QCD is not straightforward and requires a systematic treatment of the factorization. Only recently the first QCD result in the field has been reported in a short letter [22]. Below we present a detailed account of this analysis.

The paper is organized as follows. In the next section we use a simple example of quark scattering by a scalar color-singlet gluon field operator to outline the method and to derive the factorization formula for the mass-suppressed double-logarithmic corrections. In section 3 we apply the method to the analysis of the Higgs boson production in gluon fusion mediated by a bottom quark loop. In section 4 we derive the asymptotic behaviour of the leading power corrections to various massive quark form factors. The universality of our solution for different amplitudes and gauge models as well as the phenomenological applications are discussed in section 5 .

\section{Massive quark scattering by a gluon field operator}

Throughout this paper we deal with a massive quark scattering by various external currents. To introduce the main idea of our approach we start with an amplitude $\mathcal{G}$ for the scattering of a quark of mass $m_{q}$, initial momentum $p_{1}$ and final momentum $p_{2}$, by a local operator $\left(G_{\mu \nu}^{a}\right)^{2}$ of the gauge field strength tensor. The origin of such a vertex is not relevant for our discussion and one may suggest that it describes the gluon field interaction to the Higgs boson mediated by an infinitely heavy quark loop. This rather artificial amplitude is a perfect example to reveal the main features of the general problem in the most illustrative way and with minimal technical complications.

\subsection{The leading-order amplitude}

We consider the limit of the on-shell quark $p_{1}^{2}=p_{2}^{2}=m_{q}^{2}$ and the large Euclidean momentum transfer $Q^{2}=-\left(p_{2}-p_{1}\right)^{2}$ when the ratio $\rho \equiv m_{q}^{2} / Q^{2}$ is positive and small. In the light-cone coordinates $p_{1} \approx p_{1}^{-}$and $p_{2} \approx p_{2}^{+}$. The leading-order scattering is given by the one-loop diagram in figure 1 (a). Conservation of helicity at high energy requires a helicity flip on the virtual quark line. As a consequence at high energy the amplitude is suppressed by the first power of $m_{q}$. The virtual quark propagator then can be approximated as follows $S(l) \approx \frac{m_{q}}{l^{2}-m_{q}^{2}}$. Thus, the one-loop integral reduces to

$$
\frac{2 i Q^{2}}{\pi^{2}} \int \frac{d^{4} l}{\left(l^{2}-m_{q}^{2}\right)\left(p_{1}+l\right)^{2}\left(p_{2}+l\right)^{2}},
$$

where the prefactor is introduce for convenience. For the soft quark momentum $m_{q} \ll$ $l \ll Q$ the gauge boson propagators are eikonal i.e. proportional to $\frac{1}{2 p_{i} l}$, and the integral has the double-logarithmic scaling. To evaluate the double logarithmic contribution the 


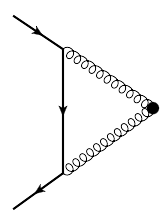

(a)

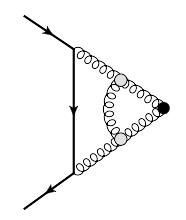

(b)

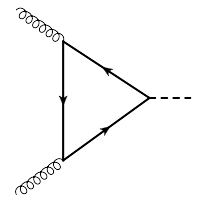

(c)

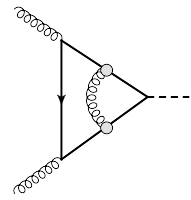

(d)

Figure 1. The leading-order one-loop Feynman diagrams for (a) quark scattering by the $\left(G_{\mu \nu}^{a}\right)^{2}$ vertex (black circle) and (c) the Higgs boson production in gluon fusion. The diagrams (b) and (d) with the effective vertices (gray circles) defined in the text represent the non-Sudakov doublelogarithmic corrections to the process (a) and (c), respectively.

propagators can be approximated as follows [1]

$$
\begin{aligned}
& \frac{1}{l^{2}-m_{q}^{2}} \approx-i \pi \delta\left(Q^{2} u v+l_{\perp}^{2}-m_{q}^{2}\right), \\
& \frac{1}{\left(p_{1}+l\right)^{2}} \approx \frac{1}{Q^{2} v}, \\
& \frac{1}{\left(p_{2}+l\right)^{2}} \approx \frac{1}{Q^{2} u},
\end{aligned}
$$

where we introduce the standard Sudakov parametrization of the soft quark momentum $l=u p_{1}+v p_{2}+l_{\perp}$. The validity of the eikonal approximation in eq. (2.2) requires $|u|,|v|<1$ and the additional kinematical constraints $u v>\rho$ has to be imposed to ensure that the soft quark propagator can go on the mass shell. After integrating eq. (2.1) over $l_{\perp}$ with the double-logarithmic accuracy we get

$$
2 \int_{\rho}^{1} \frac{\mathrm{d} v}{v} \int_{\rho / v}^{1} \frac{\mathrm{d} u}{u}=2 \ln ^{2} \rho \int_{0}^{1} \mathrm{~d} \xi \int_{0}^{1-\xi} \mathrm{d} \eta=\ln ^{2} \rho
$$

where the normalized $\operatorname{logarithmic}$ variables read $\eta=\ln v / \ln \rho, \xi=\ln u / \ln \rho$. This defines the leading order amplitude

$$
\mathcal{G}^{0}=2 C_{F} x m_{q} \bar{q} q
$$

where $x=\frac{\alpha_{s}}{4 \pi} \ln ^{2} \rho$ is a double logarithmic variable, $C_{F}=\left(N_{c}^{2}-1\right) /\left(2 N_{c}\right)$ is the quadratic Casimir operator of the fundamental representation of the $\mathrm{SU}\left(N_{c}\right)$ color group, and $\alpha_{s}$ is the strong coupling constant. Thus we have a typical situation when a soft quark exchange generates the double-logarithmic contribution to the mass-suppressed amplitude. As we see, the emission of the soft quark results in the change of the color group representation of a particle propagating along the eikonal line, or the eikonal color charge nonconservation. This is a crucial feature of the process which plays an important role in further analysis.

\subsection{Factorization of the double-logarithmic corrections}

We start with the two-loop radiative corrections. In a covariant gauge the two-loop doublelogarithmic contributions are produced by the Feynman diagrams in figure 2. Let us consider first the abelian case of the photon interaction corresponding to the diagrams in figures $2(\mathrm{a}, \mathrm{b})$. The key idea of our approach is to move the soft photon vertex from 
the virtual soft quark line in figure 2(a) to an eikonal photon line through a sequence of identities graphically represented in figure 3. Let us describe this sequence in more detail. In a covariant gauge only $A^{-}$light-cone component of the photon field can be emitted by the eikonal quark line with the momentum $p_{2}$, while the emission of the $A^{+}$ and transverse components is suppressed. Since $A^{-}$is not a physical polarization its interaction to the quark line is completely determined by gauge invariance. For the soft quark line in figure $3(\mathrm{a})$ we therefore can write the following relation

$$
S(l) \gamma^{\mu} S\left(l+l_{g}\right) \approx S(l) \gamma^{-} S\left(l+l_{g}^{+}\right)=\frac{1}{l_{g}^{+}}\left(S(l)-S\left(l+l_{g}^{+}\right)\right),
$$

with $l_{g}$ being the soft photon momentum. Multiplying eq. (2.5) with $l_{g_{\mu}} \approx l_{g_{-}}=l_{g}^{+}$ gives the standard QED Ward identity. Note that we neglect $l_{g}^{-}=l_{g_{+}}$in $S\left(l+l_{g}\right)$ to get the logarithmic scaling of the integral over $l_{g}^{-}$since the lower eikonal quark propagator is proportional to $1 / l_{g}^{-}$. The right hand side of eq. (2.5) corresponds to the diagram figure $2(\mathrm{~b})$ where the crossed circle on the quark propagator represents the replacement $S(l) \rightarrow S(l)-$ $S\left(l+l_{g}^{+}\right)$and the $1 / l_{g}^{+}$factor is absorbed into the upper eikonal quark propagator. By the momentum shift $l \rightarrow l-l_{g}^{+}$in the second term of the above expression the crossed circle can be moved to the upper eikonal photon line which becomes $\frac{1}{2 p_{1} l}-\frac{1}{2 p_{1}\left(l+l_{g}^{+}\right)}$, figure 2(c). The opposite eikonal line is not sensitive to this shift since $p_{2}^{-} \approx 0$. On the final step we use the "inverted Ward identity" on the upper eikonal photon line

$$
\frac{1}{l_{g}^{+}}\left(\frac{1}{2 p_{1} l}-\frac{1}{2 p_{1}\left(l+l_{g}^{+}\right)}\right)=\frac{1}{2 p_{1} l} 2 p_{1}-\frac{1}{2 p_{1}\left(l+l_{g}^{+}\right)} \approx \frac{1}{\left(p_{1}+l\right)^{2}} 2 p_{1}^{\mu} \frac{1}{\left(p_{1}+l+l_{g}\right)^{2}}
$$

to transform the diagram figure $2(\mathrm{c})$ into figure $2(\mathrm{~d})$ with an effective dipole coupling $2 e_{q} p_{1}^{\mu}$ to the eikonal photon, where $e_{q}$ is the quark charge. Note that we can replace $2 p_{1}\left(l+l_{g}^{+}\right)$ by $\left(p_{1}+l+l_{g}\right)^{2}$ in the gauge boson propagator as long as $l_{g} \ll Q$ since $p_{1}^{+} \approx 0$.

By adding the symmetric diagram we get a "ladder" structure characteristic to the standard eikonal factorization for the Sudakov form factor. This factorization, however, requires the summation over all possible insertions of the soft photon vertex along each eikonal line while in the case under consideration the diagram in figure 1(b) with the soft exchange between the photon lines is missing. This diagram can be added to complete the factorization and then subtracted. Note that the first Ward identity of the sequence in figure 1 is sufficient to prove the factorization of the soft photons with the momentum $l_{g} \ll m_{q}$ as it has been done in the original paper [40]. This algorithm however does not work for the momentum interval $m_{q} \ll l_{g} \ll Q$ which does contribute to the doublelogarithmic corrections. Our method extends the factorization to this region at the expense of introducing the above subtraction term, which compensates the charge variation of the eikonal line after the soft quark emission.

After adding the diagram figure 1(b) the integral over the soft photon momentum in the double-logarithmic approximation factors out with respect to the leading order amplitude and reads

$$
-\frac{e_{q}^{2}}{(4 \pi)^{2}} \frac{2 i Q^{2}}{\pi^{2}} \int \frac{d^{d} l_{g}}{l^{2}\left(\left(p_{1}+l_{g}\right)^{2}-m_{q}^{2}\right)\left(\left(p_{2}+l_{g}\right)^{2}-m_{q}^{2}\right)}=-\frac{e_{q}^{2}}{(4 \pi)^{2}}\left(2 \frac{\ln \rho}{\varepsilon}+\ln ^{2} \rho\right),
$$




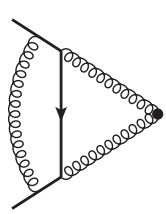

(a)

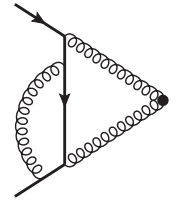

(b)

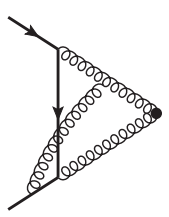

(c)

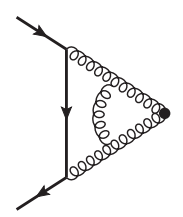

(d)

Figure 2. The two-loop Feynman diagrams for the quark scattering by the $\left(G_{\mu \nu}^{a}\right)^{2}$ vertex (black circle) which contribute in the double-logarithmic approximation. Symmetric diagrams are not shown.

where the dimensional regularization with $d=4-2 \varepsilon$ is used to deal with the infrared divergence. The above equation coincides with the one-loop on-shell Sudakov form factor, which includes all the universal Sudakov double logarithms for the amplitudes with the quark and antiquark external lines. The remaining soft photon contribution is given by the diagram figure $1(\mathrm{~b})$ with the coefficient $-e_{q}^{2}$. The corresponding two-loop integral reads

$$
\left(\frac{2 i Q^{2}}{\pi^{2}}\right)^{2} \int \frac{d^{4} l}{\left(l^{2}-m_{q}^{2}\right)\left(p_{1}+l\right)^{2}\left(p_{2}+l\right)^{2}} \frac{d^{4} l_{g}}{l_{g}^{2}\left(p_{1}+l_{g}+l\right)^{2}\left(p_{2}+l_{g}+l\right)^{2}} .
$$

The integration over the soft quark momentum $l$ is double-logarithmic if the latter can be neglected in the eikonal propagators with the soft gluon momentum $l_{g}$. This defines the conditions $l p_{1}<l_{g} p_{1}, l p_{2}<l_{g} p_{2}$ corresponding to the ordering of the Sudakov parameters along the eikonal lines $v<v_{g}, u<u_{g}$. Then in the double-logarithmic approximation the propagators take the following form

$$
\begin{aligned}
& \frac{1}{l_{g}^{2}} \approx-i \pi \delta\left(Q^{2} u_{g} v_{g}+l_{g \perp}^{2}\right), \quad \frac{1}{\left(p_{1}+l_{g}+l\right)^{2}} \approx \frac{1}{Q^{2} v_{g}}, \quad \frac{1}{\left(p_{2}+l_{g}+l\right)^{2}} \approx \frac{1}{Q^{2} u_{g}}, \\
& \frac{1}{l^{2}-m_{q}^{2}} \approx-i \pi \delta\left(Q^{2} u v+l_{\perp}^{2}-m_{q}^{2}\right), \frac{1}{\left(p_{1}+l\right)^{2}} \approx \frac{1}{Q^{2} v}, \quad \frac{1}{\left(p_{2}+l\right)^{2}} \approx \frac{1}{Q^{2} u} .
\end{aligned}
$$

After integrating over the transverse momentum components eq. (2.8) reduces to

$$
4 \int_{\rho}^{1} \frac{\mathrm{d} v}{v} \int_{\rho / v}^{1} \frac{\mathrm{d} u}{u} \int_{v}^{1} \frac{\mathrm{d} v_{g}}{v_{g}} \int_{u}^{1} \frac{\mathrm{d} u_{g}}{u_{g}} .
$$

By subsequent integrating over the parameters $u_{g}, v_{g}$ and converting the result to the logarithmic variables we get the two-loop non-Sudakov double-logarithmic correction to the amplitude

$$
\frac{e_{q}^{2}}{(4 \pi)^{2}} \ln ^{2} \rho\left(2 \int_{0}^{1} \mathrm{~d} \xi \int_{0}^{1-\xi} \mathrm{d} \eta(2 \eta \xi)\right) \mathcal{G}^{0}=\frac{e_{q}^{2}}{(4 \pi)^{2}} \frac{\ln ^{2} \rho}{6} \mathcal{G}^{0} .
$$

Note that the result is infrared finite since the quark mass regulates both collinear and soft divergences.

The above result can be generalized to QCD in a straightforward way. The difference with respect to the abelian case is that the factor $e_{q}^{2} /(4 \pi)$ should be replaced by $C_{F} \alpha_{s}$. 


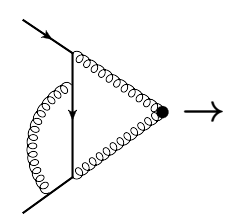

(a)

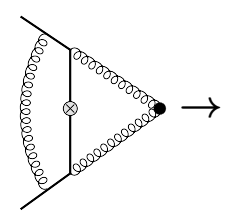

(b)

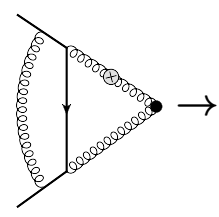

(c)

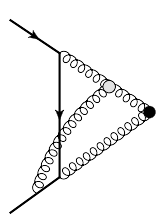

(d)

Figure 3. Diagramatic representation of the sequence of identities which move the soft gauge boson vertex from the soft quark to the eikonal gauge boson line, as explained in the text.

Moreover, the contribution similar to figure 1(b) does exist in QCD due to gluon selfcoupling and is proportional to the quadratic Casimir operator of the adjoint representation $C_{A}=N_{c}$. Thus the part of the soft gluon exchange which does not factorize into external lines is given by the diagram figure 1 (b) with the color factor $C_{A}-C_{F}$, which directly links it to the variation of the color charge along the eikonal lines. Let us now demonstrate how the above factorization emerges in the direct evaluation of the two-loop QCD diagrams in figure 2. In general the calculation can be performed in the same way as in eq. (2.8) up to the treatment of the infrared divergences not regulated by the quark mass. Figure 2(a) is the only diagram with such a divergence in the final result. The integration over the soft gluon momentum $l_{g}$ in this diagram is double-logarithmic when one can neglect it in the eikonal propagators with the soft quark momentum $l$. This defines the conditions $l_{g} p_{i} \ll l p_{i}$ corresponding to the ordering of the Sudakov parameters $v_{g} \ll v, u_{g} \ll u$. Thus $l_{g}$ should be retained only in the propagators without the soft quark momenta and the integral over the soft gluon momentum is reduced to

$$
\frac{2 i Q^{2}}{\pi^{2}} \int \frac{d^{4} l_{g}}{l_{g}^{2}\left(\left(p_{1}+l_{g}\right)^{2}-m_{q}^{2}\right)\left(\left(p_{2}+l_{g}\right)^{2}-m_{q}^{2}\right)},
$$

with the above restriction on $l_{g}$. In the double-logarithmic approximation the propagators in this expression take a form slightly different from eq. (2.9)

$$
\begin{aligned}
\frac{1}{l_{g}^{2}} & \approx-i \pi \delta\left(Q^{2} u_{g} v_{g}+l_{g_{\perp}}^{2}\right), \\
\frac{1}{\left(p_{1}+l_{g}\right)^{2}-m_{q}^{2}} & \approx \frac{1}{Q^{2}\left(v_{g}+2 \rho u_{g}\right)}, \\
\frac{1}{\left(p_{2}+l_{g}\right)^{2}-m_{q}^{2}} & \approx \frac{1}{Q^{2}\left(u_{g}+2 \rho v_{g}\right)} .
\end{aligned}
$$

After integrating eq. (2.12) over $l_{g_{\perp}}$ with the double-logarithmic accuracy we get

$$
2 \int_{\rho u_{g}}^{v} \frac{\mathrm{d} v_{g}}{v_{g}} \int_{\rho v_{g}}^{u} \frac{\mathrm{d} u_{g}}{u_{g}} .
$$

Eq. (2.14) has soft divergence when $v_{g}$ and $u_{g}$ simultaneously become small. This divergence can be removed by subtracting the factorized expression

$$
2 \int_{\rho u_{g}}^{1} \frac{\mathrm{d} v_{g}}{v_{g}} \int_{\rho v_{g}}^{1} \frac{\mathrm{d} u_{g}}{u_{g}} .
$$


The subtraction term does not depend on the soft quark momenta and is equivalent to the factorized one-loop Sudakov form factor in eq. (2.7), which does not have a nonabelian contribution. The subtracted expression reads

$$
\begin{aligned}
-2\left(\int_{v}^{1} \frac{\mathrm{d} v_{g}}{v_{g}} \int_{\rho v_{g}}^{u} \frac{\mathrm{d} u_{g}}{u_{g}}+\int_{\rho u_{g}}^{v} \frac{\mathrm{d} v_{g}}{v_{g}} \int_{u}^{1} \frac{\mathrm{d} u_{g}}{u_{g}}\right. & \left.+\int_{v}^{1} \frac{\mathrm{d} v_{g}}{v_{g}} \int_{u}^{1} \frac{\mathrm{d} u_{g}}{u_{g}}\right) \\
& =-\ln ^{2} \rho\left((\eta-\xi)^{2}+2(\eta+\xi)\right) .
\end{aligned}
$$

The contributions of the infrared subtracted diagram figure 2(a) along with the remaining infrared finite diagrams can be written as the integral over the soft quark momentum variables

$$
x\left(2 \sum_{i} c_{\lambda}^{(1)} \int_{0}^{1} \mathrm{~d} \xi \int_{0}^{1-\xi} \mathrm{d} \eta w_{\lambda}^{(1)}(\eta, \xi)\right) \mathcal{G}^{0},
$$

where the color factors $c_{\lambda}^{(1)}$ and the weight function $w_{\lambda}^{(1)}$ resulting from the logarithmic integration over the soft gluon momentum are collected in table $1 .^{1}$ Summing up the contributions we get

$$
-z\left(2 \int_{0}^{1} \mathrm{~d} \xi \int_{0}^{1-\xi} \mathrm{d} \eta(2 \eta \xi)\right) \mathcal{G}^{0}=-\frac{z}{6} \mathcal{G}^{0},
$$

where $z=\left(C_{A}-C_{F}\right) x$, which coincides with eq. (2.11) up to the modification of the effective coupling discussed above. Thus we observe the relations between the diagrams imposed by the Ward identities at the integrand level. The only new relation with respect to the abelian case provides the cancellation of the color space commutator of the soft gluon vertex at the first step of the sequence in figure 3 by the diagram figure 2(c) with the three-gluon coupling, as can be seen from the second and the third lines of table 1 . It is equivalent to the standard Ward identity for the factorization of the soft gluon emission which provides the cancellation of the nonabelian contribution in the double-logarithmic Sudakov form factor. We have verified the above result diagram by diagram through the explicit evaluation of the two-loop integrals in the high-energy limit within the expansion by regions framework [41-43].

\subsection{Resummation of the double-logarithmic corrections and the asymptotic behavior of the amplitude}

With the established factorization structure at hand it is straightforward to perform the resummation of the double logarithmic corrections to all orders of perturbation theory. Indeed, the emission of the soft gluons from an eikonal line of a given color charge factorizes and exponentiates [44] so we can apply the procedure discussed in the previous section to an arbitrary number of gluons emitted from the soft quark line. Then the factorized one-loop Sudakov logarithms exponentiate to the universal factor for the quark-antiquark external on-shell lines

$$
Z_{q}^{2}=\exp \left[-C_{F}\left(\frac{\alpha_{s}}{2 \pi} \frac{\ln \rho}{\varepsilon}+x\right)\right] .
$$

\footnotetext{
${ }^{1}$ Further details of the soft gluon momentum integration can be found in ref. [21].
} 


\begin{tabular}{|c|c|c|}
\hline$\lambda$ & $w_{\lambda}^{(1)}$ & $c_{\lambda}^{(1)}$ \\
\hline $\mathrm{a}$ & $(\eta-\xi)^{2}+2(\eta+\xi)$ & $C_{F}$ \\
$\mathrm{~b}$ & $\eta^{2}-2 \eta \xi+2 \eta$ & $-C_{F}+\frac{1}{2} C_{A}$ \\
$\mathrm{c}$ & $\eta^{2}-2 \eta \xi+2 \eta$ & $-\frac{1}{2} C_{A}$ \\
$\mathrm{~d}$ & $2 \eta \xi$ & $-C_{A}$ \\
\hline
\end{tabular}

Table 1. The weights $w_{\lambda}^{(1)}$ and the color factors $c_{\lambda}^{(1)}$ for the diagrams in figure 2. The weights for the symmetric diagrams are obtained by interchanging the $\eta$ and $\xi$ variables. The singular part of the infrared divergent diagram (a) is subtracted as discussed in the text.

The same statement is true for the soft gluon exchange between the effective vertices, figure 1(b). Thus the all-order non-Sudakov double logarithms can be obtained by replacing the one-loop contribution $-2 z \eta \xi$ in eq. (2.11) with its exponent inside the integral over the logarithmic variables. Hence the the all-order expression for the double-logarithmic corrections to the amplitude reads

$$
\mathcal{G}=Z_{q}^{2} g(-z) \mathcal{G}^{(0)}
$$

where the function $g(-z)$ incorporates the non-Sudakov contribution of figure 1(b) with an arbitrary number of the effective soft gluon exchanges. The function $g(z)$ of the variable $z=\left(C_{A}-C_{F}\right) x$ is normalized to $g(0)=1$ and is given by the two-fold integral

$$
g(z)=2 \int_{0}^{1} \mathrm{~d} \xi \int_{0}^{1-\xi} \mathrm{d} \eta e^{2 z \eta \xi} .
$$

The integral eq. (2.21) can be solved in terms of the generalized hypergeometric function

$$
g(z)={ }_{2} F_{2}(1,1 ; 3 / 2,2 ; z / 2)=2 \sum_{0}^{\infty} \frac{n !}{(2 n+2) !}(2 z)^{n}
$$

with the following asymptotic behavior at $z \rightarrow \infty$

$$
g(-z) \sim \frac{\ln (2 z)+\gamma_{E}}{z}, \quad g(z) \sim\left(\frac{2 \pi e^{z}}{z^{3}}\right)^{1 / 2},
$$

where $\gamma_{E}=0.577215 \ldots$ is the Euler constant and both limits are necessary since the variable $z$ is positive in QCD and negative in QED. The above equations determine the amplitude $\mathcal{G}$ in the high-energy limit in double-logarithmic approximation. In the next section we verify the perturbative expansion of eq. (2.20) to $\mathcal{O}\left(\alpha_{s}^{3}\right)$ by explicit evaluation of the three-loop double-logarithmic term.

\subsection{Explicit evaluation of the three-loop amplitude}

For the calculation of the three-loop logarithmic corrections we use the same method of Sudakov parameters but now have to integrate over two virtual soft gluon momenta $l_{g_{1}}$ and $l_{g_{2}}$. The relevant Feynman diagrams are given in figure 4. The sum of all the diagrams 


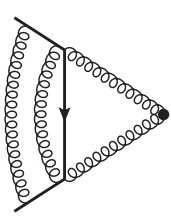

(a)

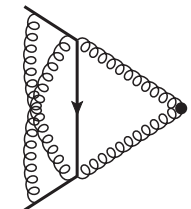

(b)

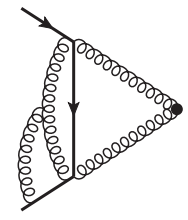

(c)

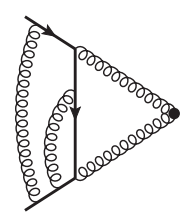

(d)

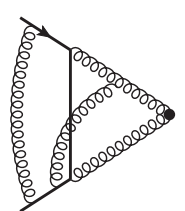

(e)

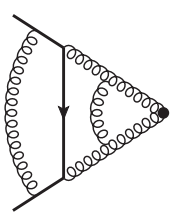

(f)

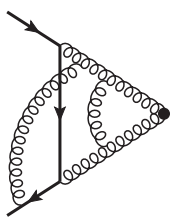

(k)

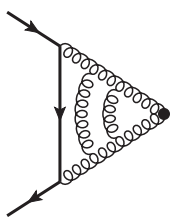

(p)

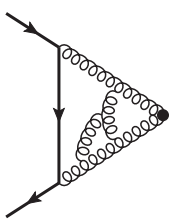

(u)

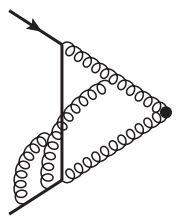

(z)

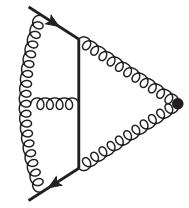

(g)

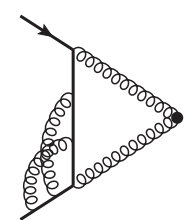

(l)

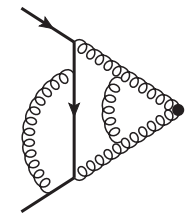

(q)

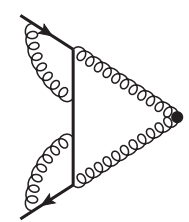

(v)

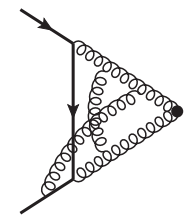

( $)$

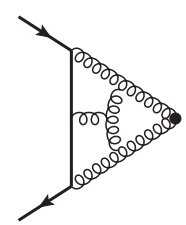

(h)

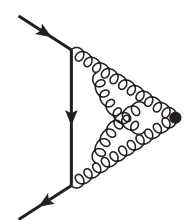

(m)

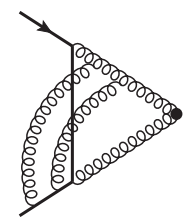

(r)

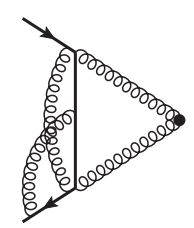

(w)

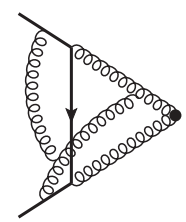

$(\sigma)$

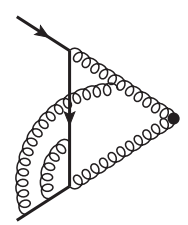

(i)

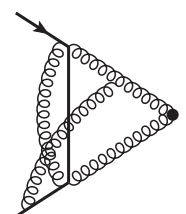

(n)

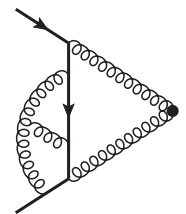

(s)

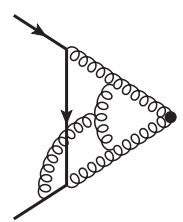

(x)

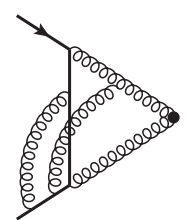

$(\tau)$

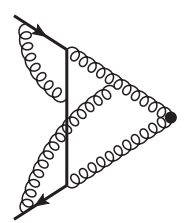

(j)

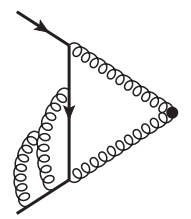

(o)

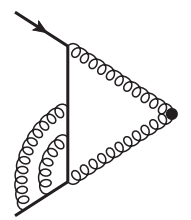

(t)

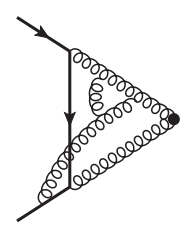

(y)

Figure 4. The three-loop Feynman diagrams for the quark scattering by the $\left(G_{\mu \nu}^{a}\right)^{2}$ vertex which contribute in the double-logarithmic approximation. Symmetric diagrams are not shown. 


\begin{tabular}{|c|c|c|}
\hline$\lambda$ & $w_{\lambda}^{(2)}$ & $c_{\lambda}^{(2)}$ \\
\hline $\mathrm{a}$ & $\begin{array}{c}2 \xi / 3+\xi^{2}+\xi^{3} / 2+\xi^{4} / 24+2 \eta / 3-\xi^{2} \eta / 2-\xi^{3} \eta / 6 \\
+\eta^{2}-\xi \eta^{2} / 2+\xi^{2} \eta^{2} / 4+\eta^{3} / 2-\xi \eta^{3} / 6+\eta^{4} / 24\end{array}$ & $4 C_{F}^{2}$ \\
\hline $\mathrm{b}$ & $\begin{array}{c}-2 \xi / 3-\xi^{2} / 2+\xi^{4} / 12-2 \eta / 3+\xi \eta-\xi^{3} \eta / 3-\eta^{2} / 2 \\
+\xi^{2} \eta^{2} / 2-\xi \eta^{3} / 3+\eta^{4} / 12\end{array}$ & $4 C_{F}^{2}-2 C_{F} C_{A}$ \\
\hline $\mathrm{c}$ & $\begin{array}{c}-2 \xi / 3-\xi^{2} / 2+\xi^{4} / 12-\eta^{2} / 4+\xi \eta^{2} / 2-\xi^{2} \eta^{2} / 4-\eta^{3} / 6 \\
+\xi \eta^{3} / 6\end{array}$ & $C_{F} C_{A}$ \\
\hline $\mathrm{d}$ & $\begin{array}{c}-2 \eta / 3+\xi^{2} \eta / 2+\xi^{3} \eta / 6-5 \eta^{2} / 4+\xi \eta^{2}-\xi^{2} \eta^{2} / 2-5 \eta^{3} / 6 \\
+\xi \eta^{3} / 2-\eta^{4} / 8\end{array}$ & $4 C_{F}^{2}-2 C_{F} C_{A}$ \\
\hline e & $\begin{array}{c}-2 \xi / 3-5 \xi^{2} / 4-5 \xi^{3} / 6-\xi^{4} / 8+\xi^{2} \eta+\xi^{3} \eta / 2 \\
+\xi \eta^{2} / 2-\xi^{2} \eta^{2} / 2+\xi \eta^{3} / 6\end{array}$ & $2 C_{F} C_{A}$ \\
\hline $\mathrm{f}$ & $-\xi^{2} \eta-\xi^{3} \eta / 2-\xi \eta^{2}+\xi^{2} \eta^{2}-\xi \eta^{3} / 2$ & $4 C_{F} C_{A}$ \\
\hline $\mathrm{g}$ & $\begin{array}{c}2 \eta / 3-\xi \eta+\xi^{3} \eta / 3+\eta^{2} / 4+\xi \eta^{2} / 2-3 \xi^{2} \eta^{2} / 4-\eta^{3} / 6 \\
+\xi \eta^{3} / 2-\eta^{4} / 8+2 \xi / 3+\xi^{2} / 4-\xi^{3} / 6-\xi^{4} / 8-\xi \eta \\
+\xi^{2} \eta / 2+\xi^{3} \eta / 2-3 \xi^{2} \eta^{2} / 4+\xi \eta^{3} / 3\end{array}$ & $C_{F} C_{A}-\frac{1}{2} C_{A}^{2}$ \\
\hline h & 0 & $\frac{1}{4} C_{A}^{2}$ \\
\hline $\mathrm{i}$ & $\eta^{2} / 2-\xi \eta^{2}+\xi^{2} \eta^{2} / 2+\eta^{3} / 2-\xi \eta^{3} / 2+\eta^{4} / 8$ & $2 C_{F} C_{A}-C_{A}^{2}$ \\
\hline j & $\xi \eta-\xi^{2} \eta / 2-\xi^{3} \eta / 2-\xi \eta^{2} / 2+5 \xi^{2} \eta^{2} / 4-\xi \eta^{3} / 2$ & $2 C_{F} C_{A}-C_{A}^{2}$ \\
\hline $\mathrm{k}$ & $\xi \eta^{2} / 2-\xi^{2} \eta^{2} / 2+\xi \eta^{3} / 3$ & $2 C_{A}^{2}$ \\
\hline 1 & $\eta^{2} / 4-\xi \eta^{2} / 2+\xi^{2} \eta^{2} / 4+\eta^{3} / 6-\xi \eta^{3} / 6+\eta^{4} / 24$ & $4 C_{F}^{2}-6 C_{F} C_{A}+2 C_{A}^{2}$ \\
\hline $\mathrm{m}$ & $\xi^{2} \eta^{2} / 4$ & $C_{A}^{2}$ \\
\hline $\mathrm{n}$ & $\begin{array}{c}2 \xi / 3+\xi^{2} / 4-\xi^{3} / 6-\xi^{4} / 8-\xi \eta+\xi^{2} \eta / 2+\xi^{3} \eta / 2 \\
-3 \xi^{2} \eta^{2} / 4+\xi \eta^{3} / 3\end{array}$ & $2 C_{F} C_{A}-C_{A}^{2}$ \\
\hline o & $\eta^{2} / 4-\xi \eta^{2} / 2+\xi^{2} \eta^{2} / 4+\eta^{3} / 6-\xi \eta^{3} / 6$ & $C_{F} C_{A}-\frac{1}{2} C_{A}^{2}$ \\
\hline $\mathrm{p}$ & $\xi^{2} \eta^{2} / 4$ & $4 C_{A}^{2}$ \\
\hline q & $\xi \eta^{2}-\xi^{2} \eta^{2}+\xi \eta^{3} / 2$ & $4 C_{F} C_{A}-2 C_{A}^{2}$ \\
\hline $\mathrm{r}$ & $\eta^{2} / 4-\xi \eta^{2} / 2+\xi^{2} \eta^{2} / 4+\eta^{3} / 3-\xi \eta^{3} / 3+\eta^{4} / 12$ & $C_{A}^{2}$ \\
\hline s & $\eta^{2} / 4-\xi \eta^{2} / 2+\xi^{2} \eta^{2} / 4+\eta^{3} / 6-\xi \eta^{3} / 6+\eta^{4} / 24$ & $C_{F} C_{A}-\frac{1}{2} C_{A}^{2}$ \\
\hline $\mathrm{t}$ & $\eta^{2} / 4-\xi \eta^{2} / 2+\xi^{2} \eta^{2} / 4+\eta^{3} / 3-\xi \eta^{3} / 3+\eta^{4} / 12$ & $4 C_{F}^{2}-4 C_{F} C_{A}+C_{A}^{2}$ \\
\hline $\mathrm{u}$ & $\xi^{2} \eta^{2} / 4$ & $C_{A}^{2}$ \\
\hline $\mathrm{v}$ & $\xi \eta-\xi^{2} \eta / 2-\xi^{3} \eta / 2-\xi \eta^{2} / 2+5 \xi^{2} \eta^{2} / 4-\xi \eta^{3} / 2$ & $4 C_{F}^{2}-4 C_{F} C_{A}+C_{A}^{2}$ \\
\hline $\mathrm{w}$ & $\begin{array}{c}2 \eta / 3-\xi \eta+\xi^{3} \eta / 3+\eta^{2} / 4+\xi \eta^{2} / 2-3 \xi^{2} \eta^{2} / 4-\eta^{3} / 6 \\
+\xi \eta^{3} / 2-\eta^{4} / 8\end{array}$ & $4 C_{F}^{2}-4 C_{F} C_{A}+C_{A}^{2}$ \\
\hline $\mathrm{x}$ & $\xi \eta^{2} / 2-\xi^{2} \eta^{2} / 2+\xi \eta^{3} / 6$ & $\frac{1}{2} C_{A}^{2}$ \\
\hline $\mathrm{y}$ & $\eta^{2} / 4-\xi^{2} \eta^{2} / 4+\eta^{3} / 6+\eta^{4} / 24$ & $\frac{1}{2} C_{A}^{2}$ \\
\hline $\mathrm{z}$ & $\eta^{2} / 4-\xi \eta^{2} / 2+\xi^{2} \eta^{2} / 4+\eta^{3} / 6-\xi \eta^{3} / 6$ & $\frac{1}{2} C_{A}^{2}$ \\
\hline$\rho$ & $\xi^{2} \eta / 2+\xi^{3} \eta / 6-\xi^{2} \eta^{2} / 2$ & $C_{A}^{2}$ \\
\hline$\sigma$ & $2 \xi / 3+\xi^{2} / 4-\xi^{3} / 6-\xi^{4} / 8-\xi \eta^{2} / 2+\xi^{2} \eta^{2} / 2-\xi \eta^{3} / 6$ & $\frac{1}{2} C_{A}^{2}$ \\
\hline$\tau$ & $\eta^{2} / 2-\xi \eta^{2}+\xi^{2} \eta^{2} / 2+\eta^{3} / 2-\xi \eta^{3} / 2+\eta^{4} / 8$ & $2 C_{F} C_{A}-C_{A}^{2}$ \\
\hline
\end{tabular}

Table 2. The weights $w_{\lambda}^{(2)}$ and the color factors $c_{\lambda}^{(2)}$ for the diagrams in figure 4 . 
has the infrared divergent part described by the Sudakov factor eq. (2.19). As in the twoloop case the divergent parts of the individual diagrams can be separated in the Sudakov parameter space by subtracting the factorized infrared divergent contributions where the upper integration for a given parameter $v_{g_{i}}$ or $u_{g_{j}}$ is set to 1 (cf. eq. (2.15)). The factorized double-pole singular contributions of the diagrams in figures $4(\mathrm{a}-\mathrm{c})$ indeed add up to

$$
\frac{C_{F}^{2}}{2}\left(\frac{\alpha_{s}}{2 \pi} \frac{\ln \rho}{\varepsilon}+x\right)^{2} \mathcal{G}^{0}
$$

while the factorized single-pole contributions of the diagrams in figures $4(\mathrm{a}-\mathrm{f})$ give

$$
\frac{C_{F} z}{6}\left(\frac{\alpha_{s}}{2 \pi} \frac{\ln \rho}{\varepsilon}+x\right) \mathcal{G}^{0}
$$

which agrees with eq. (2.19)-(2.22). Though some of the remaining diagrams taken separately are infrared divergent, their sum is finite and gives the following contribution to the amplitude

$$
x^{2}\left(2 \sum_{\lambda} c_{\lambda}^{(2)} \int_{0}^{1} \mathrm{~d} \xi \int_{0}^{1-\xi} \mathrm{d} \eta w_{\lambda}^{(2)}(\eta, \xi)\right) \mathcal{G}^{0},
$$

where the color factors $c_{\lambda}^{(2)}$ and the weight function $w_{\lambda}^{(2)}$ resulting from the logarithmic integration over the two soft gluon momenta are collected in table 2. Note that the weights $w_{\lambda}^{(2)}$ correspond to the infrared subtracted diagrams and the weights for the symmetric diagrams not shown in figure 4 are obtained by interchanging the $\eta$ and $\xi$ variables and should be included into the sum. It is straightforward to check that the sum in eq. (2.26) reduces to

$$
z^{2}\left(2 \int_{0}^{1} \mathrm{~d} \xi \int_{0}^{1-\xi} \mathrm{d} \eta 2(\eta \xi)^{2}\right) \mathcal{G}^{0}=\frac{z^{2}}{45} \mathcal{G}^{0},
$$

in full agreement with eqs. (2.20)-(2.22).

\section{Higgs boson production mediated by bottom quark loop}

The analysis and the result of the previous section can be generalized in a straightforward way to an important case of the bottom quark mediated Higgs boson production in gluon fusion. We postpone the discussion of the phenomenological aspects of this process to the last section and focus now on the structure of the radiative corrections. The leading order contribution is given by the one-loop diagram in figure 1(c). Note that the dominant contribution to the gluon fusion process is given by the same diagram with the top quark loop and in the formal limit of the large top quark mass $m_{t} \gg m_{H}$ is proportional to the square of the Higgs boson mass $m_{H}$. By contrast for the intermediate bottom quark with $m_{b} \ll m_{H}$ the amplitude is suppressed by the square of the bottom quark mass. Indeed, the Higgs boson coupling to the bottom quark is proportional to $m_{b}$. Then the scalar interaction of the Higgs boson results in a helicity flip at the interaction vertex and helicity conservation requires the amplitude to vanish in the limit $m_{b} \rightarrow 0$ even if the Higgs coupling to the bottom quark is kept fixed. As in the example considered in the 


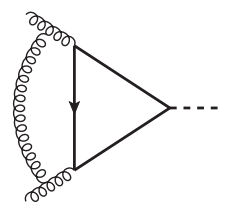

(a)

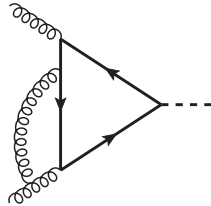

(b)

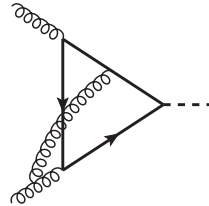

(c)

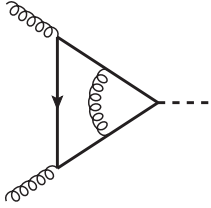

(d)

Figure 5. The two-loop Feynman diagrams for the bottom quark mediated $g g \rightarrow H$ fusion, which contribute in the double-logarithmic approximation. Symmetric diagrams and the diagrams with the opposite direction of the closed quark line are not shown.

previous section the additional power of $m_{b}$ originates from the $t$-channel quark propagator which effectively becomes scalar and results in double-logarithmic scaling of the diagram absent for the top quark contribution. By using the explicit one-loop result the bottom quark mediated amplitude can be written in such a way that its power suppression and the logarithmic enhancement is manifest

$$
\mathcal{M}_{g g \rightarrow H}^{b(0)}=-\frac{3}{2} \ln ^{2} \rho \rho \mathcal{M}_{g g \rightarrow H}^{t(0)},
$$

where $\rho=m_{b}^{2} / s$ is now a Minkowskian parameter, $s \approx m_{H}^{2}$ is the total energy of colliding gluons, and the result is given in terms of the heavy top quark mediated amplitude $\mathcal{M}_{g g \rightarrow H}^{t(0)}$, which corresponds to a local gluon-gluon-Higgs interaction vertex and has one independent helicity component.

Thus the processes in figures $1(\mathrm{a}, \mathrm{c})$ are similar in a few important aspects: they are mass suppressed due to the quark helicity flip, the double-logarithmic contribution is induced by the soft quark exchange and the color charge is not conserved along the eikonal lines. Moreover, since the eikonal or Wilson lines are characterized by the momentum and color charge but not the spin, in the double-logarithmic approximation the processes are identical up to the color group representation of the external lines and the direction of the color charge flow to/from an eikonal line at the soft quark emission vertex. Therefore the factorization structure of the double-logarithmic corrections found in the section 2 directly applies to the case under consideration. In particular the two-loop non-Sudakov double-logarithmic contribution is given by the diagram figure $1(\mathrm{~d})$ where the effective gluon exchange has the color weight $C_{F}-C_{A}$ rather than $C_{A}-C_{F}$ of the diagram figure 1(b), and the higher-order non-Sudakov double-logarithmic terms are described by the same function $g(z)$ with $C_{A}$ and $C_{F}$ exchanged, i.e. with the opposite sign of the argument. Hence to all orders in $\alpha_{s}$ we get

$$
\mathcal{M}_{g g \rightarrow H}^{b}=Z_{g}^{2} g(z) \mathcal{M}_{g g \rightarrow H}^{b(0)},
$$

where

$$
Z_{g}^{2}=\exp \left[-\frac{C_{A} s^{-\varepsilon}}{\varepsilon^{2}} \frac{\alpha_{s}}{2 \pi}\right]
$$

is the Sudakov factor for a gluon scattering. Let us now demonstrate how the above factorization is realized for the two-loop corrections. The relevant diagrams are given in 


\begin{tabular}{|c|c|c|}
\hline$\lambda$ & $\tilde{w}_{\lambda}^{(1)}$ & $c_{\lambda}^{(1)}$ \\
\hline $\mathrm{a}$ & $-(\eta+\xi-\tau)^{2}$ & $\frac{1}{2} C_{A}$ \\
$\mathrm{~b}$ & $\eta^{2}+2 \eta \xi-2 \eta \tau$ & $\frac{1}{4} C_{A}$ \\
$\mathrm{c}$ & $\eta^{2}+2 \eta \xi-2 \eta \tau$ & $\frac{1}{4} C_{A}$ \\
$\mathrm{~d}$ & $2 \eta \xi$ & $-\frac{1}{2} C_{F}$ \\
\hline
\end{tabular}

Table 3. The weights $\tilde{w}_{\lambda}^{(1)}$ and the color factors $c_{\lambda}^{(1)}$ for the diagrams in figure 5.

figure 5 and the corresponding contribution to the amplitude can be written in the form similar to eq. (2.17)

$$
x\left(2 \sum_{i} c_{\lambda}^{(1)} \int_{0}^{1} \mathrm{~d} \xi \int_{0}^{1-\xi} \mathrm{d} \eta \tilde{w}_{\lambda}^{(1)}(\eta, \xi)\right) \mathcal{M}_{g g \rightarrow H}^{b(0)},
$$

with the color factors $c_{\lambda}^{(1)}$ and the weights $\tilde{w}_{\lambda}^{(1)}$ collected in table 3 . As before the weights for the symmetric diagrams not shown in figure 5 are obtained by interchanging the $\eta$ and $\xi$ variables and should be included into the sum. To make the factorization of the Sudakov logarithms explicit we do not subtract the factorized contribution and the functions $\tilde{w}_{i}^{(1)}$ correspond to the unsubtracted Feynman integrals over the soft gluon momentum, which are infrared divergent. They are regularized by introducing a small auxiliary gluon mass $\lambda_{g} \ll m_{b}$, which is more convenient for the calculation in the Sudakov parameter space than dimensional regularization. In table 3 the dependence on the infrared regulator is encoded into the parameter $\tau=\ln ^{2}\left(\lambda_{g}^{2} / s\right) / \ln ^{2}\left(m_{b}^{2} / s\right)$. The contributions of the individual diagrams in eq. (3.4) combine into the sum of two terms

$$
\left(2 \int_{0}^{1} \mathrm{~d} \xi \int_{0}^{1-\xi} \mathrm{d} \eta\left(2 z \eta \xi-C_{A} x \tau\right)\right) \mathcal{M}_{g g \rightarrow H}^{b(0)} .
$$

The first term in eq. (3.4) coincides with the expression for the diagram figure 1(d) and represents the first-order term in the perturbative expansion of the function $g(z)$. The second term in the brackets does not depend on the soft quark momentum variables, i.e. here the soft gluon momentum integral factorizes and gives the one-loop massive gluon Sudakov form factor $-C_{A} x \tau=-\frac{C_{A} \alpha_{s}}{4 \pi} \ln ^{2}\left(\lambda_{g}^{2} / s\right)$. After converting to the dimensional regularization it recovers the first-order term in the perturbative expansion of $Z_{g}^{2}$. Thus the double-logarithmic contributions factorize at the integrand level as suggested by the Ward identities discussed in the previous section. Note that the two-loop contribution to eq. (3.2) agrees with the analytical result for the amplitude with an arbitrary value of the quark mass [45] expanded in the series in $\rho$.

\section{Quark form factors beyond the leading-power approximation}

In this section we consider the asymptotic behavior of the leading mass-suppressed contribution to the amplitude of quark scattering in an external field. The problem is more 


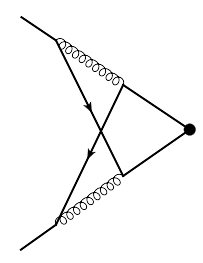

(a)

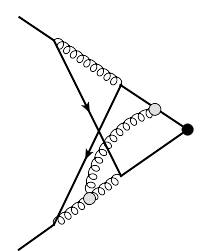

(b)

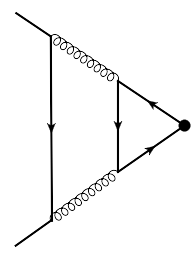

(c)

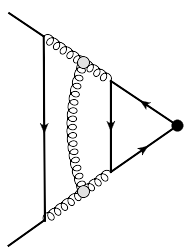

(d)

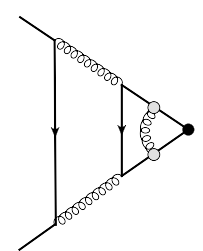

(e)

Figure 6. The leading order two-loop Feynman diagrams for (a) vector form factor $F_{1}^{(1)}$ and (c) scalar form factor $F_{S}^{(1)}$ in the double-logarithmic approximation. The diagrams with an effective soft gluon exchange which incorporate the non-Sudakov double-logarithmic corrections to (b) vector and $(\mathrm{d}, \mathrm{e})$ scalar form factor. Symmetric diagrams and the diagrams with the opposite direction of the closed quark line are not shown.

complex since in contrast to the amplitudes considered in the previous sections the quark form factors do get the leading power contribution which does not vanish in $m_{q} \rightarrow 0$ limit. In refs. $[14,17]$ it was shown within the expansion by regions framework that a soft gauge boson exchange responsible for the standard Sudakov logarithms does not generate the leading mass suppressed double-logarithmic contribution. Such a contribution results from the soft fermion pair exchange between the eikonal lines. Therefore the approach elaborated in the previous section can be naturally extended to the quark form factors. We start with the analysis of the external vector field and consider the scalar field case next.

\subsection{Vector form factor}

The amplitude of a quark scattering in an external color-singlet vector field can be parameterized in the standard way by the Dirac and Pauli form factors. The Pauli form factor contribution to the amplitude at high energy is suppressed by the first power of $\rho$ but does not acquire the double logarithmic corrections in the approximation discussed in this paper. Indeed, the leading-order one-loop Pauli form factor $F_{2}$ is finite so the higher-order Sudakov double logarithms will give a subleading contribution to the scattering amplitude suppressed by an additional power of the coupling constant. Thus we focus on the high-energy behavior of the Dirac form-factor $F_{1}$ described by an asymptotic series in $\rho$

$$
F_{1}=Z_{q}^{2} \sum_{n=0}^{\infty} \rho^{n} F_{1}^{(n)},
$$

where $F_{1}^{(n)}$ are given by the power series in $\alpha_{s}$ with the coefficients depending on $\rho$ only logarithmically, and we use the same notations and kinematics as in section 2. Since the Sudakov corrections in eq. (4.1) are factored out, in the double-logarithmic approximation the leading term of the expansion is just the Born value $F_{1}^{(0)}=1$, and the double-logarithmic corrections to the leading power-suppressed term $F_{1}^{(1)}$ are purely non-Sudakov. According to the results of refs. $[14,21]$ such corrections are induced by the nonplanar soft quark pair exchange, figure 6(a), and start with the two-loop contribution. In contrast to the previously considered cases the vector interaction conserves helicity and require a helicity flip on each of soft quark lines which become sufficiently singular to develop the double 


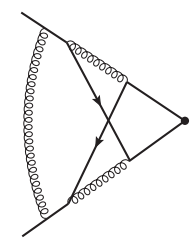

(a)

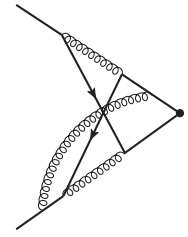

(d)

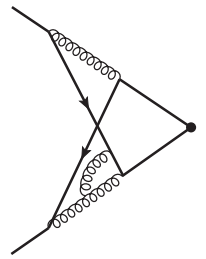

$(\mathrm{g})$

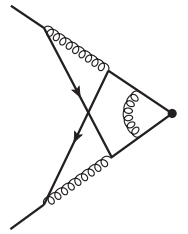

(b)

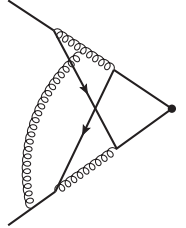

(e)

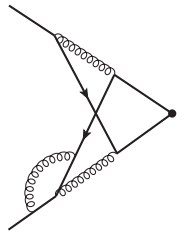

(h)

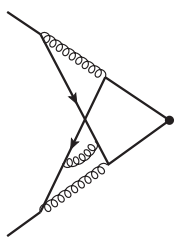

(c)

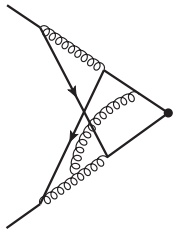

(f)

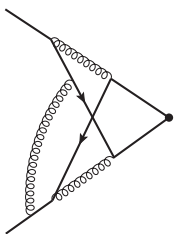

(i)

Figure 7. The three-loop diagrams contributing to the vector form factor $F_{1}^{(1)}$ in the doublelogarithmic approximation. Symmetric diagrams are not shown. The remaining diagrams either do not have the double-logarithmic integration region or have vanishing color factor.

logarithmic scaling. The corresponding Feynman integral reads

$$
\begin{aligned}
\left(\frac{2 i Q^{2}}{\pi^{2}}\right)^{2} \int\left(\frac{d^{4} l_{1}}{\left(l_{1}^{2}-m_{q}^{2}\right)\left(p_{2}+l_{1}\right)^{2}\left(\left(p_{1}+l_{1}+l_{2}\right)^{2}-m_{q}^{2}\right)}\right. & \\
& \left.\times \frac{d^{4} l_{2}}{\left(l_{2}^{2}-m_{q}^{2}\right)\left(p_{1}+l_{2}\right)^{2}\left(\left(p_{2}+l_{1}+l_{2}\right)^{2}-m_{q}^{2}\right)}\right) .
\end{aligned}
$$

The integration over the soft quark momenta $l_{i}$ in this case is double-logarithmic when $l_{2} p_{1}<l_{1} p_{1}, l_{1} p_{2}<l_{2} p_{2}$ and the corresponding Sudakov parameters are ordered along the eikonal lines $v_{2}<v_{1}, u_{1}<u_{2}$. With the additional kinematical constraints $u_{i} v_{i}>\rho$ the integral over the Sudakov parameters reduces to

$$
4 \ln ^{4} \rho \int_{0}^{1} \mathrm{~d} \eta_{1} \int_{\eta_{1}}^{1} \mathrm{~d} \eta_{2} \int_{0}^{1-\eta_{2}} \mathrm{~d} \xi_{2} \int_{\xi_{2}}^{1-\eta_{1}} \mathrm{~d} \xi_{1}=\frac{\ln ^{4} \rho}{3}
$$

which corresponds to the leading-order result

$$
\left[F_{1}^{(1)}\right]_{2-\text { loop }}=\frac{C_{F}\left(C_{A}-2 C_{F}\right)}{6} x^{2},
$$

in agreement with [46]. To derive the factorization formula for the higher-order doublelogarithmic terms let us consider the three-loop corrections, figure 7, and start with the abelian QED case. Following the algorithm described in section 2.2 we use the sequence of 


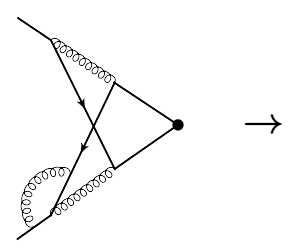

(a)

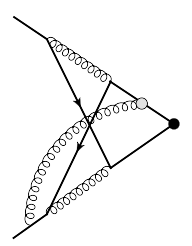

(b)

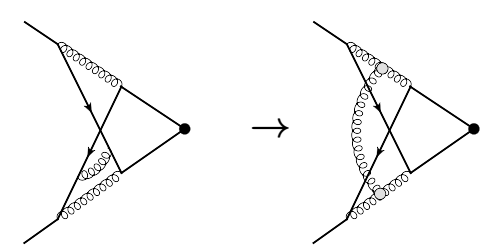

(c)

(d)

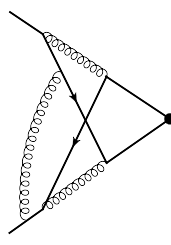

(e)

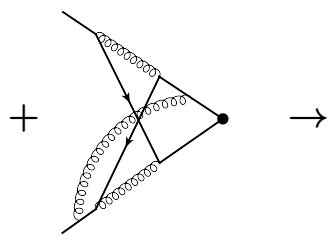

(f)

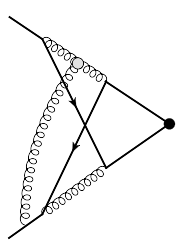

$(\mathrm{g})$

Figure 8. Diagramatic representation of the transformation which moves the soft gauge boson vertex from a soft quark to an eikonal line.

Ward identities and the soft quark momentum shifts to move the soft photon vertex from a soft quark to an eikonal line. The result of the transformation is shown in figure 8 , where the effective vertex is proportional to the charge of the corresponding external line. The first two relations are rather straightforward to derive. In the third relation the presence of the additional diagram figure $8(\mathrm{f})$ needs to be clarified. First we note that this diagram has an opposite sign with respect to figure 8 (b) since the photon couples to the antiquark rather than the incoming quark line. Thus the diagram figure $8(\mathrm{f})$ is not relevant for the eikonal factorization of the soft photon ladder. On the contrary, its role is similar to the diagram figure 2(c) which cancels the color space commutator when the soft gluon vertex is moved in figure 2(b). Though now we consider the abelian case, such commutator appears in the transformation of figure $8(\mathrm{e})$ since the electric charge is not conserved along the eikonal line. A set of the resulting ladder diagrams figures $8(\mathrm{~b}, \mathrm{~d}, \mathrm{~g})$ together with figures $7(\mathrm{a}, \mathrm{b})$ needs the last missing permutation of the soft photon vertex, figure 6(b), to complete the eikonal factorization. After adding this diagram the soft photon exchange factorizes into the one-loop Sudakov factor in eq. (4.1). Thus the remaining soft photon contribution to $F_{1}^{(1)}$ is given by the negative of figure $6(\mathrm{~b})$, and the symmetric one. A characteristic feature of the diagram figure $6(\mathrm{~b})$ is that the soft gluon connects two eikonal lines determined by the same soft quark momentum. Such a diagram cannot be obtained by the transformation of another diagram described in section 2.2 and has to be added by hand to complete the Sudakov logarithms factorization. Hence the above property can be a guiding principle for selecting the diagrams which define the non-Sudakov double logarithmic contribution. As in the example discussed in section 2.2 the QCD result is obtained from the QED one by substituting $-e_{q}^{2} /(4 \pi)$ with $\left(C_{A}-C_{F}\right) \alpha_{s}$ in the above diagram. In analogy with eq. (2.17) we can write the three-loop contribution to the form factor as follows

$$
x\left(12 \sum_{i} c_{\lambda}^{(1)} \int_{0}^{1} \mathrm{~d} \eta_{1} \int_{\eta_{1}}^{1} \mathrm{~d} \eta_{2} \int_{0}^{1-\eta_{2}} \mathrm{~d} \xi_{2} \int_{\xi_{2}}^{1-\eta_{1}} \mathrm{~d} \xi_{1} w_{\lambda}^{(1)}(\eta, \xi)\right)\left[F_{1}^{(1)}\right]_{2-\text { loop }}
$$




\begin{tabular}{|c|c|c|}
\hline$\lambda$ & $w_{\lambda}^{(1)}$ & $c_{\lambda}^{(1)}$ \\
\hline $\mathrm{a}$ & $-\eta_{2}\left(\eta_{2}+2\right)-\xi_{1}\left(\xi_{1}-2 \eta_{2}+2\right)$ & $-C_{F}$ \\
$\mathrm{~b}$ & $2 \xi_{2} \eta_{1}$ & $-C_{F}$ \\
$\mathrm{c}$ & $2\left(\xi_{1}-\xi_{2}\right)\left(\eta_{2}-\eta_{1}\right)$ & $C_{A}-C_{F}$ \\
$\mathrm{~d}$ & $-\eta_{1}\left(\eta_{1}-2 \xi_{1}+2\right)$ & $C_{A}-C_{F}$ \\
$\mathrm{e}$ & $\left(\eta_{2}-\eta_{1}\right)\left(\eta_{1}+\eta_{2}-2 \xi_{1}+2\right)$ & $-\frac{C_{A}}{2}$ \\
$\mathrm{f}$ & $2 \eta_{1}\left(\xi_{1}-\xi_{2}\right)$ & $-\frac{C_{A}}{2}$ \\
$\mathrm{~g}$ & $2 \eta_{2}\left(\xi_{1}-\xi_{2}\right)$ & $-\frac{C_{A}}{2}$ \\
$\mathrm{~h}$ & $\eta_{1}\left(\eta_{1}-2 \xi_{1}+2\right)$ & $\frac{C_{A}}{2}-C_{F}$ \\
$\mathrm{i}$ & $\eta_{2}\left(\eta_{2}-2 \xi_{1}+2\right)$ & $\frac{C_{A}}{2}-C_{F}$ \\
\hline
\end{tabular}

Table 4. The weights $w_{\lambda}^{(1)}$ and the color factors $c_{\lambda}^{(1)}$ for the diagrams in figure 7 .

where $c_{\lambda}^{(1)}$ and $w_{\lambda}^{(1)}$ are listed in table 4 . The weights for the symmetric diagrams not shown in figure 7 are obtained in this case by the replacement $\eta_{1} \leftrightarrow \xi_{2}$ and $\eta_{2} \leftrightarrow \xi_{1}$. Eq. (4.5) sums up to

$$
-z\left(12 \int_{0}^{1} \mathrm{~d} \eta_{1} \int_{\eta_{1}}^{1} \mathrm{~d} \eta_{2} \int_{0}^{1-\eta_{2}} \mathrm{~d} \xi_{2} \int_{\xi_{2}}^{1-\eta_{1}} \mathrm{~d} \xi_{1}\left(2 \eta_{1}\left(\xi_{1}-\xi_{2}\right)+2 \xi_{2}\left(\eta_{2}-\eta_{1}\right)\right)\right)\left[F_{1}^{(1)}\right]_{2-\text { loop }},
$$

which can be recognized as the contribution of the effective soft gluon exchange in figure $6(\mathrm{~b})$ and in the symmetric diagram.

Now the factorization formula for the leading power-suppressed contribution to the vector form factor can be written as follows

$$
F_{1}^{(1)}=\frac{C_{F}\left(C_{A}-2 C_{F}\right)}{6} x^{2} f(-z),
$$

where the function $f(-z)$ incorporates the non-Sudakov contribution of figure 6(b) with an arbitrary number of the effective soft gluon exchanges and is normalized to the two-loop result $f(0)=1$. This function is obtained by exponentiating the single effective soft gluon exchange in eq. (4.6) and therefore has the following integral representation

$$
f(z)=12 \int_{0}^{1} \mathrm{~d} \eta_{1} \int_{\eta_{1}}^{1} \mathrm{~d} \eta_{2} \int_{0}^{1-\eta_{2}} \mathrm{~d} \xi_{2} \int_{\xi_{2}}^{1-\eta_{1}} \mathrm{~d} \xi_{1} e^{2 z \eta_{1}\left(\xi_{1}-\xi_{2}\right)} e^{2 z \xi_{2}\left(\eta_{2}-\eta_{1}\right)} .
$$

It is difficult to solve the four-fold integral eq. (4.8) in a closed analytic form. However, the coefficients of the series $f(z)=1+\sum_{n=1}^{\infty} c_{n} z^{n}$ can be computed for any given $n$ corresponding to the $(n+2)$-loop double-logarithmic contribution and have the following large- $n$ behavior $c_{n} \sim \frac{\ln n}{n ! 2^{n} n^{5 / 2}}$. The first ten coefficients of the series are listed in table 5 . The asymptotic behavior of the function at $z \rightarrow \infty$ reads

$$
f(-z) \sim C_{-}\left(\frac{\ln z}{z}\right)^{2}, \quad f(z) \sim C_{+} \ln z\left(\frac{e^{z}}{z^{5}}\right)^{1 / 2}
$$




\begin{tabular}{|c|c|c|c|c|c|c|c|c|c|c|}
\hline$n$ & 1 & 2 & 3 & 4 & 5 & 6 & 7 & 8 & 9 & 10 \\
\hline $2^{n} n^{2} n ! c_{n}$ & $\frac{2}{5}$ & $\frac{88}{105}$ & $\frac{8}{7}$ & $\frac{70144}{51975}$ & $\frac{640}{429}$ & $\frac{25344}{15925}$ & $\frac{2727424}{1640925}$ & $\frac{1868824576}{1091215125}$ & $\frac{8994816}{5143775}$ & $\frac{274430420480}{15460335891}$ \\
\hline
\end{tabular}

Table 5. The normalized coefficients of the Taylor series for the function $f(z)$, eq. (4.8), up to $n=10$.

where the constant $C_{-}=3.6 \ldots, C_{+}=14.8 \ldots$ are found numerically. The result eq. (4.7) vanishes for $N_{c} \rightarrow \infty$, which is consistent with the explicit evaluation of the three-loop massive form factor in this limit [47].

\subsection{Scalar form factor}

The quark scattering in the external scalar field is parametrized by a single form factor $F_{S}$ which has the high-energy asymptotic expansion similar to eq. (4.1)

$$
F_{S}=Z_{q}^{2} \sum_{n=0}^{\infty} \rho^{n} F_{S}^{(n)}
$$

with the Born result normalized to $F_{S}^{(0)}=1$. The general arguments of the previous section on the origin of the leading mass suppressed double-logarithmic corrections are equally applicable to the scalar form factor. However, the contribution of the nonplanar diagram figure 6(a) in this case vanishes. Indeed, the scalar vertex induces an additional helicity flip along the quark line and requires an odd number of the soft quark exchanges. At the same time the planar soft quark pair exchange figure 6(c) with a closed quark line, which vanishes for the external vector field by Furry theorem, does contribute in the scalar case. The relevant Feynman integral reads

$$
\begin{aligned}
& \left(\frac{2 i Q^{2}}{\pi^{2}}\right)^{2} \int\left(\frac{d^{4} l_{1}}{\left(l_{1}^{2}-m_{q}^{2}\right)\left(p_{2}+l_{1}\right)^{2}\left(p_{1}+l_{1}\right)^{2}}\right. \\
& \left.\times \frac{d^{4} l_{2}}{\left(l_{2}^{2}-m_{q}^{2}\right)\left(\left(p_{1}+l_{1}+l_{2}\right)^{2}-m_{q}^{2}\right)\left(\left(p_{2}+l_{1}+l_{2}\right)^{2}-m_{q}^{2}\right)}\right),
\end{aligned}
$$

with the double-logarithmic integration region $l_{1} p_{1}<l_{2} p_{1}, l_{1} p_{2}<l_{2} p_{2}$, or $v_{1}<v_{2}, u_{1}<u_{2}$. It reduces to

$$
4 \ln ^{4} \rho \int_{0}^{1} \mathrm{~d} \eta_{1} \int_{0}^{1-\eta_{1}} \mathrm{~d} \xi_{1} \int_{\eta_{1}}^{1-\xi_{1}} \mathrm{~d} \eta_{2} \int_{\xi_{1}}^{1-\eta_{2}} \mathrm{~d} \xi_{2}=\frac{\ln ^{4} \rho}{6}
$$

corresponding to the leading-order form factor

$$
\left[F_{S}^{(1)}\right]_{2-\text { loop }}=-\frac{C_{F} T_{F}}{3} x^{2}
$$

where $T_{F}=1 / 2$, in agreement with [48]. Let us now discuss the factorization of the double-logarithmic corrections. By using the same procedure as for the vector form factor one can reduce the non-Sudakov part of the corrections to the contribution of the diagrams in figures $6(\mathrm{~d}, \mathrm{e})$ with the effective soft gluon exchange between the eikonal lines determined 


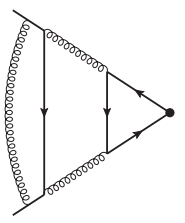

(a)

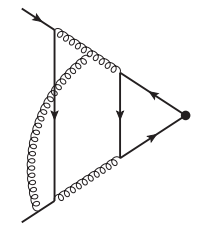

(e)

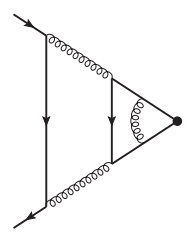

(b)

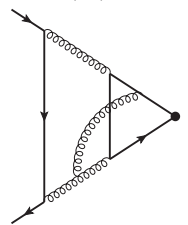

(f)

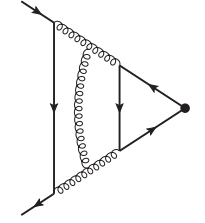

(c)

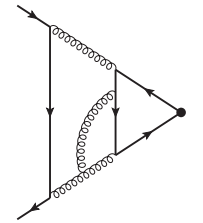

(g)

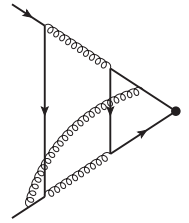

(d)

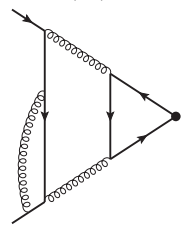

(h)

Figure 9. The three-loop diagrams contributing to the scalar form factor $F_{S}^{(1)}$. Symmetric diagrams and the diagrams with the opposite direction of the closed quark line are not shown. The remaining diagrams either do not have the double-logarithmic integration region or have vanishing color factor.

by the same soft quark momentum. The diagram figure $6(\mathrm{~d})$ has the color factor $C_{A}-C_{F}$ while the diagram figure $6(\mathrm{e})$ is proportional to $C_{F}-C_{A}$ as dictated by the variation of the color charge along the eikonal lines in each case. The only subtlety is related to the fact that in the diagram figure 6(d) the soft gluon momentum integral factors out from the inner quark loop in the same way as the Sudakov corrections factor out in the $g g \rightarrow H$ amplitude discussed in section 3 . The corresponding correction to the form factor reads

$$
-z\left(24 \int_{0}^{1} \mathrm{~d} \eta_{1} \int_{0}^{1-\eta_{1}} \mathrm{~d} \xi_{1} \int_{\eta_{1}}^{1-\xi_{1}} \mathrm{~d} \eta_{2} \int_{\xi_{1}}^{1-\eta_{2}}\left(2 \eta_{2} \xi_{2}-2 \eta_{1} \xi_{1}\right)\right)\left[F_{S}^{(1)}\right]_{2-\text { loop }},
$$

where the first and the second terms in the brackets represent the contributions of the diagrams in figure 6(d) and figure 6(e), respectively.

The three-loop non-Sudakov double logarithmic corrections can of course be evaluated explicitly. The relevant three-loop diagrams are given in figure 9 and the corresponding contribution to the form factor written in the standard form reads

$$
x\left(24 \sum_{i} c_{\lambda}^{(1)} \int_{0}^{1} \mathrm{~d} \eta_{1} \int_{0}^{1-\eta_{1}} \mathrm{~d} \xi_{1} \int_{\eta_{1}}^{1-\xi_{1}} \mathrm{~d} \eta_{2} \int_{\xi_{1}}^{1-\eta_{2}} w_{\lambda}^{(1)}(\eta, \xi)\right)\left[F_{S}^{(1)}\right]_{2-\text { loop }},
$$

where $c_{\lambda}^{(1)}$ and $w_{\lambda}^{(1)}$ are listed in table 5 . The weights for the symmetric diagrams not shown in figure 9 are obtained in this case by the replacement $\eta_{1} \leftrightarrow \xi_{1}$ and $\eta_{2} \leftrightarrow \xi_{2}$. As we expect the sum in eq. (4.15) reduces to eq. (4.14) confirming the above factorization. Thus we get the following expression describing the asymptotic behavior of the leading mass-suppressed contribution to the scalar form factor

$$
F_{S}^{(1)}=-\frac{C_{F} T_{F}}{3} x^{2} f_{S}(-z),
$$

where the function

$$
f_{S}(z)=24 \int_{0}^{1} \mathrm{~d} \eta_{1} \int_{0}^{1-\eta_{1}} \mathrm{~d} \xi_{1} \int_{\eta_{1}}^{1-\xi_{1}} \mathrm{~d} \eta_{2} \int_{\xi_{1}}^{1-\eta_{2}} \mathrm{~d} \xi_{2} e^{2 z \eta_{2} \xi_{2}} e^{-2 z \eta_{1} \xi_{1}}
$$




\begin{tabular}{|c|c|c|}
\hline$\lambda$ & $w_{\lambda}^{(1)}$ & $c_{\lambda}^{(1)}$ \\
\hline $\mathrm{a}$ & $\eta_{2}\left(\eta_{2}+2\right)+\xi_{2}\left(\xi_{2}-2 \eta_{2}+2\right)$ & $C_{F}$ \\
$\mathrm{~b}$ & $-2 \xi_{1} \eta_{1}$ & $C_{F}$ \\
$\mathrm{c}$ & $2\left(\xi_{1}-\xi_{2}\right)\left(\eta_{1}-\eta_{2}\right)$ & $-C_{A}$ \\
$\mathrm{~d}$ & $\eta_{1}\left(\eta_{1}-2 \xi_{2}+2\right)$ & $-\frac{1}{2} C_{A}$ \\
$\mathrm{e}$ & $\left(\eta_{2}-\eta_{1}\right)\left(\eta_{2}+\eta_{1}-2 \xi_{2}+2\right)$ & $-\frac{1}{2} C_{A}$ \\
$\mathrm{f}$ & $2 \eta_{1}\left(\xi_{1}-\xi_{2}\right)$ & $\frac{1}{2} C_{A}$ \\
$\mathrm{~g}$ & $2 \eta_{1}\left(\xi_{1}-\xi_{2}\right)$ & $\frac{1}{2} C_{A}$ \\
$\mathrm{~h}$ & $\eta_{2}\left(\eta_{2}-2 \eta_{2} \xi_{2}+2\right)$ & $\frac{1}{2} C_{A}-C_{F}$ \\
\hline
\end{tabular}

Table 6. The weights $w_{\lambda}^{(1)}$ and the color factors $c_{\lambda}^{(1)}$ for the diagrams in figure 8 . The contributions of the diagrams with the opposite direction of the closed quark line are included.

is determined by the diagrams in figures $6(\mathrm{~d}, \mathrm{e})$ with the corresponding exponential factors given separately. Eq. (4.16) is consistent with the expansion of the exact result for the three-loop massive form factor in the large- $N_{c}$ limit [49].

Amazingly, though the topology of the diagrams in figure 6(b) and figures $6(\mathrm{~d}, \mathrm{e})$ is completely different, eqs. (4.8) and (4.17) describe the same function

$$
f_{S}(z) \equiv f(z),
$$

as it can be easily verified. It is straightforward to extend the analysis to the axial $F_{A}$ and the pseudoscalar $F_{P}$ form factors, for which we obtain the result in the form of eq. (4.7) and eq. (4.16) with $f_{A}(z)=-f(z)$ and $f_{P}(z)=f(z)$, respectively.

\section{Summary and discussion}

We have presented the details of the first systematic analysis of the high-energy asymptotic behaviour of the QCD amplitudes beyond the leading-power approximation and derived allorder double-logarithmic result for the leading mass-suppressed terms in typical two-scale problems. In contrast to the Sudakov logarithms, the mass-suppressed double-logarithmic corrections are induced by a soft quark exchange. The structure of the corrections and the asymptotic behavior of the amplitudes in this case crucially depend on the color flow in a given process and are determined by the eikonal color charge nonconservation. After separating the standard Sudakov factors the remaining non-Sudakov double-logarithmic corrections are described by two universal functions $g( \pm z)$ and $f( \pm z)$, eqs. (2.22) and (4.8), of the variable $z=\frac{\alpha_{s}}{4 \pi}\left(C_{A}-C_{F}\right) \ln ^{2}\left(m_{q}^{2} / Q^{2}\right)$ for the processes with single and double soft quark exchange, respectively. These functions play the role of "Sudakov exponent" for the non-Sudakov double-logarithmic corrections. They grow as $e^{z / 2}$ i.e. are exponentially enhanced for large positive values of the argument and are power suppressed for the large negative values. Our result reveals highly nontrivial relations between the asymptotic behavior of different amplitudes and the amplitudes in different gauge theories. In particular, 
if a QCD amplitude gets the exponential enhancement at high energy, an amplitude with the inverted color charge flow from the eikonal line defined by a scattering particle, or the same amplitude in QED are suppressed by a power of the large logarithm, ${ }^{2}$ and vice versa.

In general the amplitudes with larger number of scattering particles, such as Bhabha scattering in QED [17], get contributions from both single and double soft fermion exchange. The factorization structure in this case can be more complex and the corresponding asymptotic expressions may involve new functions besides $g(z)$ and $f(z)$.

One of the most interesting phenomenological applications of our analysis is an estimate of the high-order corrections to the bottom quark mediated Higgs boson production in gluon fusion, which is one of the main sources of uncertainty in the theoretical predictions for the Higgs cross section at the Large Hadron Collider. The effective expansion parameter in this case is $\ln ^{2}\left(m_{b}^{2} / m_{H}^{2}\right) \alpha_{s} \approx 40 \alpha_{s}$ rather than $\alpha_{s}$, and the resummation of the doublelogarithmic corrections is mandatory for a reliable theoretical estimate. From the result of section 3 we can immediately get such an estimate for the exclusive Higgs boson production cross section with a veto on the jet transverse momentum of the order of the bottom quark mass. In this case the bottom quark loop induced interaction is local with respect to the soft emission and therefore results in an overall correction factor to the leading order cross section. The dominant contribution is due to its interference with the top-loop mediated amplitudes which reads

$$
\delta \sigma_{g g \rightarrow H}=-3 \rho \ln ^{2} \rho\left(1+\frac{z}{6}+\frac{z^{2}}{45}+\frac{z^{3}}{420}+\ldots\right) \sigma_{g g \rightarrow H},
$$

where the series in $z$ is the Taylor expansion of the function $g(z)$. Up to the next-toleading order the exact dependence of the cross section on the bottom quark mass without the expansion in $\rho$ has been known for a while [50]. The next-to-next-to-leading $\mathcal{O}\left(z^{2}\right)$ term in eq. (5.1) is new. Numerically for $m_{H}=125 \mathrm{GeV}, m_{b}=5 \mathrm{GeV}$, and $\alpha_{s}\left(m_{b}\right)=0.21$ we get $z \approx 1.2$ which in general is not a good expansion parameter. However for this value of $z$ the above series becomes $1+0.19+0.030+0.0037+\ldots$ and converges sufficiently fast. The $\mathcal{O}\left(z^{2}\right)$ term results in about $0.6 \%$ decrease of the cross section, which can be considered as an estimate of the bottom quark loop effect in the next-to-next-to-leading order. An interesting and important problem is the generalization of our result for more inclusive observables such as Higgs plus jet production cross section and the Higgs boson transverse momentum distribution, which can be significantly affected by the bottom quark contribution. Only the abelian part of the corresponding double-logarithmic corrections is known so far [16].

\section{Acknowledgments}

The work of A.P. is supported in part by NSERC, Institute for Theoretical Physics, ETH Zürich, and Perimeter Institute for Theoretical Physics. The work of T.L. is supported by NSERC.

\footnotetext{
${ }^{2}$ In some cases such as a QED quark form factor this suppression can be cancelled by the energy dependence of the leading order result.
} 
Open Access. This article is distributed under the terms of the Creative Commons Attribution License (CC-BY 4.0), which permits any use, distribution and reproduction in any medium, provided the original author(s) and source are credited.

\section{References}

[1] V.V. Sudakov, Vertex parts at very high-energies in quantum electrodynamics, Sov. Phys. JETP 3 (1956) 65 [Zh. Eksp. Teor. Fiz. 30 (1956) 87] [INSPIRE].

[2] J. Frenkel and J.C. Taylor, Exponentiation of leading infrared divergences in massless Yang-Mills theories, Nucl. Phys. B 116 (1976) 185 [InSPIRE].

[3] A.V. Smilga, Next-to-leading logarithms in the high-energy asymptotics of the quark form-factor and the jet cross-section, Nucl. Phys. B 161 (1979) 449 [INSPIRE].

[4] A.H. Mueller, On the asymptotic behavior of the Sudakov form-factor, Phys. Rev. D 20 (1979) 2037 [INSPIRE].

[5] J.C. Collins, Algorithm to compute corrections to the Sudakov form-factor, Phys. Rev. D 22 (1980) 1478 [INSPIRE].

[6] A. Sen, Asymptotic behavior of the Sudakov form-factor in QCD, Phys. Rev. D 24 (1981) 3281 [INSPIRE].

[7] G.F. Sterman, Summation of large corrections to short distance hadronic cross-sections, Nucl. Phys. B 281 (1987) 310 [inSPIRE].

[8] A. Ferroglia, M. Neubert, B.D. Pecjak and L.L. Yang, Two-loop divergences of scattering amplitudes with massive partons, Phys. Rev. Lett. 103 (2009) 201601 [arXiv:0907.4791] [INSPIRE].

[9] E. Laenen, L. Magnea, G. Stavenga and C.D. White, Next-to-eikonal corrections to soft gluon radiation: a diagrammatic approach, JHEP 01 (2011) 141 [arXiv:1010.1860] [INSPIRE].

[10] A. Banfi, P.F. Monni and G. Zanderighi, Quark masses in Higgs production with a jet veto, JHEP 01 (2014) 097 [arXiv: 1308.4634] [INSPIRE].

[11] T. Becher and G. Bell, Enhanced nonperturbative effects through the collinear anomaly, Phys. Rev. Lett. 112 (2014) 182002 [arXiv:1312.5327] [INSPIRE].

[12] D. de Florian, J. Mazzitelli, S. Moch and A. Vogt, Approximate $N^{3}$ LO Higgs-boson production cross section using physical-kernel constraints, JHEP 10 (2014) 176 [arXiv: 1408.6277] [INSPIRE].

[13] C. Anastasiou et al., Higgs boson gluonffusion production beyond threshold in $N^{3} L O Q C D$, JHEP 03 (2015) 091 [arXiv: 1411.3584] [INSPIRE].

[14] A.A. Penin, High-energy limit of quantum electrodynamics beyond Sudakov approximation, Phys. Lett. B $\mathbf{7 4 5}$ (2015) 69 [Erratum ibid. B $\mathbf{7 5 1}$ (2015) 596] [arXiv:1412.0671] [INSPIRE].

[15] A.A. Almasy, N.A. Lo Presti and A. Vogt, Generalized threshold resummation in inclusive DIS and semi-inclusive electron-positron annihilation, JHEP 01 (2016) 028 [arXiv: 1511.08612] [INSPIRE].

[16] K. Melnikov and A. Penin, On the light quark mass effects in Higgs boson production in gluon fusion, JHEP 05 (2016) 172 [arXiv: 1602.09020] [INSPIRE]. 
[17] A.A. Penin and N. Zerf, Two-loop Bhabha scattering at high energy beyond leading power approximation, Phys. Lett. B 760 (2016) 816 [Erratum ibid. B 771 (2017) 637] [arXiv: 1606.06344] [INSPIRE].

[18] D. Bonocore et al., Non-abelian factorisation for next-to-leading-power threshold logarithms, JHEP 12 (2016) 121 [arXiv:1610.06842] [INSPIRE].

[19] R. Boughezal, X. Liu and F. Petriello, Power corrections in the $N$-jettiness subtraction scheme, JHEP 03 (2017) 160 [arXiv: 1612.02911] [INSPIRE].

[20] I. Moult, I.W. Stewart and G. Vita, A subleading operator basis and matching for $g g \rightarrow H$, JHEP 07 (2017) 067 [arXiv: 1703.03408] [INSPIRE].

[21] T. Liu, A.A. Penin and N. Zerf, Three-loop quark form factor at high energy: the leading mass corrections, Phys. Lett. B 771 (2017) 492 [arXiv:1705.07910] [INSPIRE].

[22] T. Liu and A.A. Penin, High-energy limit of QCD beyond the Sudakov approximation, Phys. Rev. Lett. 119 (2017) 262001 [arXiv:1709.01092] [INSPIRE].

[23] M. Beneke, M. Garny, R. Szafron and J. Wang, Anomalous dimension of subleading-power $N$-jet operators, JHEP 03 (2018) 001 [arXiv: 1712.04416] [INSPIRE].

[24] R. Boughezal, A. Isgrò and F. Petriello, Next-to-leading-logarithmic power corrections for $N$-jettiness subtraction in color-singlet production, Phys. Rev. D 97 (2018) 076006 [arXiv: 1802.00456] [INSPIRE].

[25] R. Brüser, S. Caron-Huot and J.M. Henn, Subleading Regge limit from a soft anomalous dimension, JHEP 04 (2018) 047 [arXiv: 1802.02524] [INSPIRE].

[26] I. Moult, I.W. Stewart, G. Vita and H.X. Zhu, First subleading power resummation for event shapes, JHEP 08 (2018) 013 [arXiv: 1804.04665] [INSPIRE].

[27] S. Alte, M. König and M. Neubert, Effective field theory after a new-physics discovery, JHEP 08 (2018) 095 [arXiv: 1806.01278] [INSPIRE].

[28] M. Beneke, M. Garny, R. Szafron and J. Wang, Anomalous dimension of subleading-power $N$-jet operators II, arXiv: 1808.04742 [INSPIRE].

[29] B. Feucht, J.H. Kuhn, A.A. Penin and V.A. Smirnov, Two loop Sudakov form-factor in a theory with mass gap, Phys. Rev. Lett. 93 (2004) 101802 [hep-ph/0404082] [InSPIRE].

[30] B. Jantzen, J.H. Kuhn, A.A. Penin and V.A. Smirnov, Two-loop electroweak logarithms in four-fermion processes at high energy, Nucl. Phys. B 731 (2005) 188 [Erratum ibid. B 752 (2006) 327] [hep-ph/0509157] [INSPIRE].

[31] A.A. Penin, Two-loop corrections to Bhabha scattering, Phys. Rev. Lett. 95 (2005) 010408 [hep-ph/0501120] [INSPIRE].

[32] A.A. Penin, Two-loop photonic corrections to massive Bhabha scattering, Nucl. Phys. B 734 (2006) 185 [hep-ph/0508127] [INSPIRE].

[33] R. Bonciani, A. Ferroglia and A.A. Penin, Heavy-flavor contribution to Bhabha scattering, Phys. Rev. Lett. 100 (2008) 131601 [arXiv:0710.4775] [InSPIRE].

[34] R. Bonciani, A. Ferroglia and A.A. Penin, Calculation of the two-loop heavy-flavor contribution to Bhabha scattering, JHEP 02 (2008) 080 [arXiv:0802.2215] [INSPIRE].

[35] J.H. Kühn, F. Metzler and A.A. Penin, Next-to-next-to-leading electroweak logarithms in W-pair production at ILC, Nucl. Phys. B 795 (2008) 277 [Erratum ibid. B 818 (2009) 135] [arXiv:0709.4055] [INSPIRE]. 
[36] J.H. Kuhn, F. Metzler, A.A. Penin and S. Uccirati, Next-to-next-to-leading electroweak logarithms for W-pair production at LHC, JHEP 06 (2011) 143 [arXiv:1101.2563] [INSPIRE].

[37] A.A. Penin and G. Ryan, Two-loop electroweak corrections to high energy large-angle Bhabha scattering, JHEP 11 (2011) 081 [arXiv:1112.2171] [INSPIRE].

[38] V.G. Gorshkov, V.N. Gribov, L.N. Lipatov and G.V. Frolov, Doubly logarithmic asymptotic behavior in quantum electrodynamics, Sov. J. Nucl. Phys. 6 (1968) 95 [Yad. Fiz. 6 (1967) 129] [INSPIRE].

[39] M.I. Kotsky and O.I. Yakovlev, On the resummation of double logarithms in the process Higgs $\rightarrow \gamma \gamma$, Phys. Lett. B 418 (1998) 335 [hep-ph/9708485] [INSPIRE].

[40] D.R. Yennie, S.C. Frautschi and H. Suura, The infrared divergence phenomena and high-energy processes, Annals Phys. 13 (1961) 379 [INSPIRE].

[41] M. Beneke and V.A. Smirnov, Asymptotic expansion of Feynman integrals near threshold, Nucl. Phys. B 522 (1998) 321 [hep-ph/9711391] [INSPIRE].

[42] V.A. Smirnov, Asymptotic expansions of two loop Feynman diagrams in the Sudakov limit, Phys. Lett. B 404 (1997) 101 [hep-ph/9703357] [INSPIRE].

[43] V.A. Smirnov, Applied asymptotic expansions in momenta and masses, Springer Tracts Mod. Phys. 177 (2002) 1.

[44] J. Frenkel and J.C. Taylor, Nonabelian eikonal exponentiation, Nucl. Phys. B 246 (1984) 231 [INSPIRE].

[45] C. Anastasiou, S. Beerli, S. Bucherer, A. Daleo and Z. Kunszt, Two-loop amplitudes and master integrals for the production of a Higgs boson via a massive quark and a scalar-quark loop, JHEP 01 (2007) 082 [hep-ph/0611236] [INSPIRE].

[46] W. Bernreuther et al., Two-loop QCD corrections to the heavy quark form-factors: the vector contributions, Nucl. Phys. B 706 (2005) 245 [hep-ph/0406046] [INSPIRE].

[47] J. Henn, A.V. Smirnov, V.A. Smirnov and M. Steinhauser, Massive three-loop form factor in the planar limit, JHEP 01 (2017) 074 [arXiv: 1611.07535] [INSPIRE].

[48] W. Bernreuther et al., Decays of scalar and pseudoscalar Higgs bosons into fermions: two-loop QCD corrections to the Higgs-quark-antiquark amplitude, Phys. Rev. D 72 (2005) 096002 [hep-ph/0508254] [INSPIRE].

[49] R.N. Lee, A.V. Smirnov, V.A. Smirnov and M. Steinhauser, Three-loop massive form factors: complete light-fermion and large- $N_{c}$ corrections for vector, axial-vector, scalar and pseudo-scalar currents, JHEP 05 (2018) 187 [arXiv: 1804.07310] [INSPIRE].

[50] M. Spira, A. Djouadi, D. Graudenz and P.M. Zerwas, Higgs boson production at the LHC, Nucl. Phys. B 453 (1995) 17 [hep-ph/9504378] [INSPIRE]. 